\title{
Creation of Bifurcation-Type Elastase-Induced Aneurysms in Rabbits
}

\author{
Y.H. Ding, R. Kadirvel, D. Dai, and D.F. Kallmes
}

\section{ABSTRACT}

SUMMARY: Elastase incubation was performed in the LCCA in 13 New Zealand white rabbits. Three weeks after incubation, DSA demonstrated that $10(10 / 13,77 \%)$ bifurcation-type aneurysms at the origin of the LCCA were present; mean aneurysm neck, width, and height values were $3.7 \pm 1.1,3.8 \pm 0.9$, and $8.7 \pm 2.3 \mathrm{~mm}$, respectively. The LCCA can be used to create bifurcation aneurysms in rabbits.

ABBREVIATIONS: DSA = digital subtraction angiography; IADSA = intra-arterial digital subtraction angiography; IVDSA = intravenous digital subtraction angiography; $\mathrm{LCCA}=$ left common carotid artery; RCCA = right common carotid artery

N ew Zealand white rabbits have been widely used for creation of RCCA elastase-induced aneurysms, ${ }^{1-5}$ which have several advantages over the traditional surgical aneurysms created by using anastomosis between the vein pouch and carotid artery. ${ }^{1,6-8}$ Several modifications have been made to the RCCA model to customize resultant aneurysm morphology. ${ }^{9-11}$ However, the RCCA model is of sidewall morphology, albeit along a curved vessel and, thus, is limited regarding its use in studying bifurcation-type aneurysm morphologies.

In contradistinction to the RCCA, which originates as a bifurcation with the subclavian artery, the LCCA typically originates as a trifurcation, along with the brachiocephalic artery and the aortic arch. ${ }^{3}$ In this study, we applied techniques of aneurysm creation analogous to those used for sidewall RCCA elastase-induced aneurysms to the LCCA and catalogued resultant aneurysm morphology and size.

\section{DESCRIPTION OF THE TECHNIQUE Aneurysm Creation}

Elastase-induced aneurysms were created in 13 New Zealand white rabbits, following the approval of our Institutional Animal Care and Use Committee. Anesthesia was induced with an intramuscular injection of ketamine, xylazine, and acepromazine (75,

Received July 16, 2010; accepted after revision February 17, 2011.

From the Neuroradiology Research Laboratory, Department of Radiology, Mayo Clinic, Rochester, Minnesota.

This work was supported by National Institutes of Health grant R01 NS46246.

Please address correspondence to David F. Kallmes, MD, Department of Radiology, Mayo Clinic, 200 First St SW, Rochester, MN 55905; e-mail:

kallmes.david@mayo.edu

--m Indicates open access to non-subscribers at www.ajnr.org

http://dx.doi.org/10.3174/ajnr.A2666
5 , and $1 \mathrm{mg} / \mathrm{kg}$, respectively). Using sterile technique, we exposed and isolated the LCCA. A 1- to 2-mm bevelled arteriotomy was made, and a $5 \mathrm{~F}$ vascular sheath (Avanti, Cordis Endovascular, Miami Lakes, Florida) was advanced retrogradely in the LCCA to a point approximately $3 \mathrm{~cm}$ cephalad to the origin of LCCA. A 3F Fogarty balloon (Baxter Healthcare, Irvine, California) was advanced through the sheath to the level of the origin of the LCCA with fluoroscopic guidance (Advantx, GE Healthcare, Milwaukee, Wisconsin) and was inflated with iodinated contrast material (iohexol, Omnipaque 300; GE Healthcare, Princeton, New Jersey). Porcine elastase (approximately $120 \mathrm{U} / \mathrm{mL}$; Worthington Biochemical, Lakewood, New Jersey) was incubated within the lumen of the LCCA above the inflated balloon for 20 minutes, after which the catheter, balloon, and sheath were removed and the LCCA was ligated below the sheath entry site.

\section{Imaging Follow-Up}

Three weeks following aneurysm creation, DSA was performed, 12 cases through IVDSA and 1 case by IADSA. Details of the IVDSA procedure have been reported previously. ${ }^{1}$ Briefly, $7 \mathrm{~mL}$ of iodinated contrast material (iohexol, Omnipaque 300) was injected into the left ear vein through an angiocatheter at approximately $2 \mathrm{~mL} / \mathrm{s}$. For IADSA, a right femoral artery cutdown was followed by $5 \mathrm{~F}$ catheter inserted into the aortic arch, followed by injection of $5 \mathrm{~mL}$ of contrast (Omnipaque 300). The x-ray exposure rate was 2 frames per second. ${ }^{12} 3$ D DSA was also performed in 4 cases by using the Artis Zee fluoroscopy system (Siemens, Erlangen, Germany), which involves a 5 -second $200^{\circ}$ rotation with acquisition of 133 images during $20 \mathrm{~mL}$ of contrast injection (Omnipaque 300, injection rate $4 \mathrm{~mL}$ per second) in the ascending aorta. ${ }^{13}$ Original $3 \mathrm{D}$ rotational images were reconstructed and displayed by using a volume-rendering technique. 

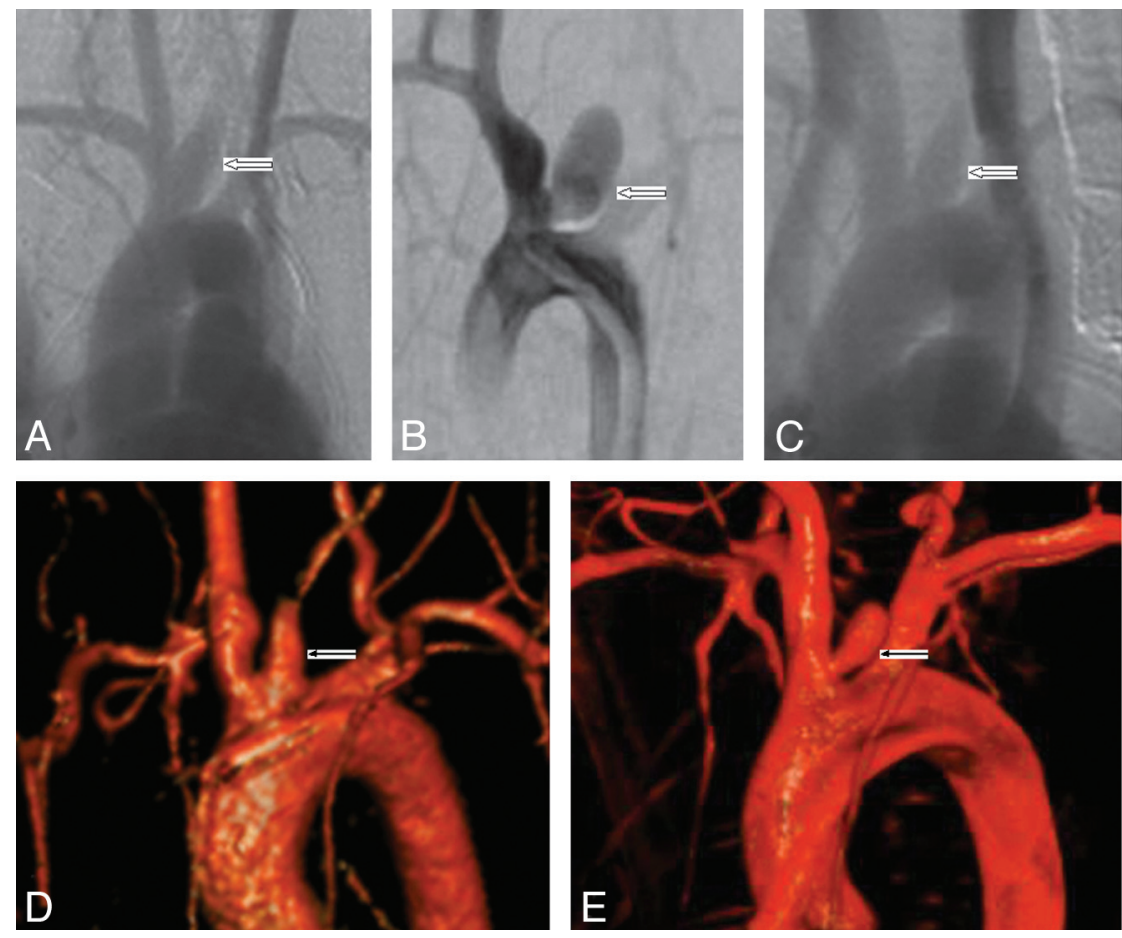

FIG 1. $A-C$, Anteroposterior IVDSA images show 3 bifurcation aneurysms (block arrow). D and E, 3D DSA images show 2 aneurysms located in the bifurcation between the aortic arch and brachiocephalic trunk (black arrow).

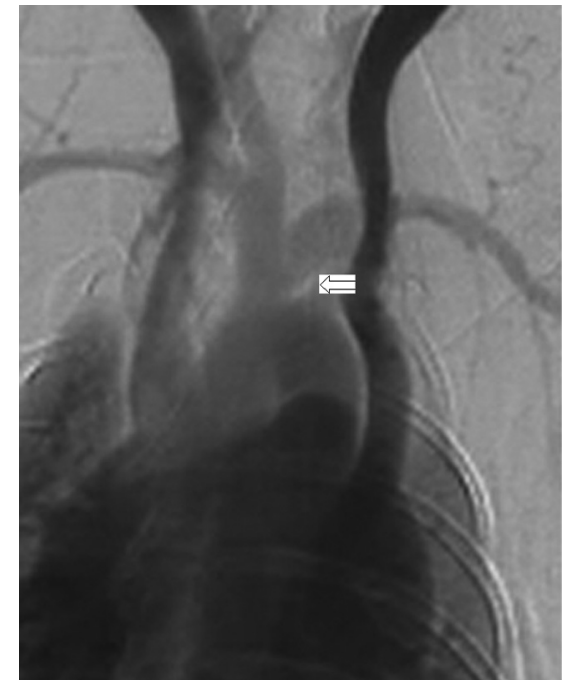

FIG 2. Anteroposterior (IVDSA) image shows a sidewall aneurysm originating from brachiocephalic trunk only (block arrow).

Patent saccular aneurysmal structures were present in all cases, with bifurcation-type aneurysm morphologies in 10 (77\%) of 13 rabbits (Fig 1). A "bifurcation-type aneurysm" was defined as an aneurysm that originated exactly from the angle between brachiocephalic trunk and the aortic arch. Two (15\%) cases showed the LCCA aneurysms originating from brachiocephalic trunk alone, with resultant sidewall aneurysm morphology (Fig 2), and 1 (8\%) sidewall aneurysm originated from the aortic arch directly (Fig 3 ).

The width, height, and neck diameters of the aneurysm cavities were determined and calculated by using IVDSA images with the external sizing device as a reference. The mean aneurysm neck

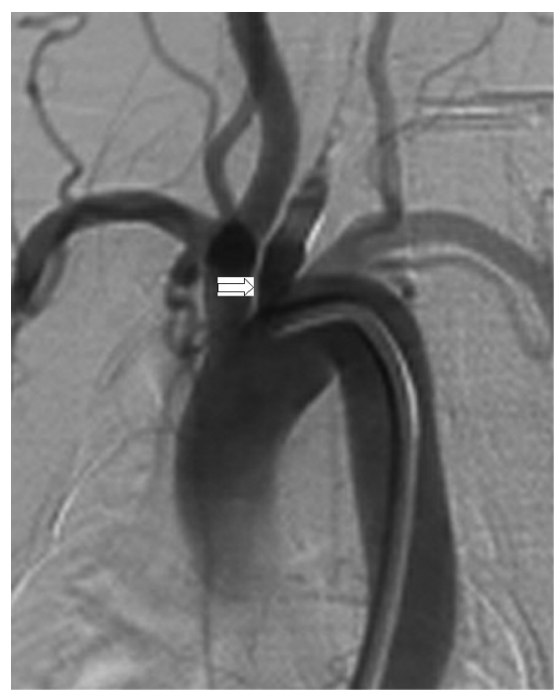

FIG 3. Anteroposterior IADSA image shows another sidewall aneurysm originating from aortic arch (block arrow).

size was $3.7 \pm 1.1 \mathrm{~mm}$ (range, $2.1-6.5 \mathrm{~mm}$ ). The mean aneurysm width was $3.8 \pm 0.9 \mathrm{~mm}$ (range, $2.6-5.9 \mathrm{~mm}$ ). The mean aneurysm height was $8.7 \pm 2.3 \mathrm{~mm}$ (range, $7-14 \mathrm{~mm}$ ).

\section{DISCUSSION}

In this study, we modified the typical rabbit elastase-induced aneurysm model by ligating and injuring with elastase the left, rather than right, common carotid artery. As a result, the large majority of resultant aneurysms demonstrated bifurcation anatomy rather than the typical sidewall morphology in the RCCA model. A small 
number of subjects showed sidewall aneurysm morphology, arising from either the brachiocephalic trunk or the aortic arch.

Bifurcation aneurysms are exposed to different hemodynamic features compared with sidewall aneurysms. ${ }^{14}$ They are more common than sidewall aneurysms in patients with intracranial aneurysms, including aneurysms that originate from the bifurcation of the internal carotid and posterior communicating arteries, and the middle cerebral artery bifurcation. Compared with the sidewall aneurysm model, the bifurcation aneurysm shows more encouraging results for evaluation of neurovascular devices. ${ }^{15,16}$ Studies involving computational fluid dynamics simulations also use the bifurcation aneurysm as an important tool. ${ }^{17}$ Thus, this study will expand the application of the elastase-induced aneurysm model to investigate the physiology of bifurcation aneurysms and test endovascular devices aimed at treating bifurcationtype aneurysms.

Elastase-induced aneurysms have previously been created from the LCCA, ${ }^{18}$ in which the aneurysms were created by using transfemoral endovascular means with distal LCCA occlusion by using detachable balloons or coils; elastase injury was achieved from an endovascular approach that was time-consuming and associated with substantial morbidity. The current study offers a practical, simple direct surgical exposure method, which is similar to that widely applied for RCCA aneurysm creation.

This study had a small sample size. Further study regarding this issue is being done to validate this model.

Disclosures: Ramanathan Kadirvel, Research Support (including provision of equipment or materials): American Heart Association, Details: Research grant. Daying Dai, Research Support (including provision of equipment or materials): animal work. David Kallmes, Research Support (including provision of equipment or materials): Nfocus, MicroVention, Micrus, Cordis, Sequent, ev3.

\section{REFERENCES}

1. Altes TA, Cloft HJ, Short JG, et al. 1999 ARRS Executive Council Award: creation of saccular aneurysms in the rabbit-a model suitable for testing endovascular devices. American Roentgen Ray Society. AJR Am J Roentgenol 2000;174:349-54

2. Krings T, Hans FJ, Moller-Hartmann W, et al. Time-of-flight, phase contrast and contrast enhanced magnetic resonance angiography for pre-interventional determination of aneurysm size, configuration, and neck morphology in an aneurysm model in rabbits. Neurosci Lett 2002;326:46-50

3. Ding YH, Dai D, Layton KF, et al. Vascular anatomic variation in rabbits. J Vasc Interv Radiol 2006;17:1031-35
4. Ding YH, Dai D, Lewis DA, et al. Angiographic and histologic analysis of experimental aneurysms embolized with platinum coils, Matrix, and HydroCoil. AJNR Am J Neuroradiol 2005;26:1757-63

5. Kallmes DF, Ding YH, Dai D, et al. A new endoluminal, flow-disrupting device for treatment of saccular aneurysms. Stroke 2007;38:2346-52

6. Dai D, Ding YH, Kallmes DF. Histopathologic and immunohistochemical comparison in human, rabbit, and swine aneurysms embolized with platinum coils. AJNR Am J Neuroradiol 2005;26:2560-68

7. Kallmes DF, Altes TA, Vincent DA, et al. Experimental side-wall aneurysms: a natural history study. Neuroradiology 1999;41:338-41

8. Byrne JV, Hope JK, Hubbard N, et al. The nature of thrombosis induced by platinum and tungsten coils in saccular aneurysms. AJNR Am J Neuroradiol 1997;18:29-33

9. Ding YH, Dai DY, Lewis DA, et al. Can neck size in elastaseinduced aneurysms be controlled? A prospective study. AJNR Am J Neuroradiol 2005;26:2364-67

10. Ding YH, Danielson MA, Kadirvel R, et al. Modified technique to create more reproducible elastase-induced aneurysms in rabbits. Neuroradiology 2006;48:528-32

11. Ding YH, Dai D, Lewis DA, et al. Control of aneurysm volume by adjusting the position of ligation during creation of elastase-induced aneurysms: a prospective study. AJNR Am J Neuroradiol 2007;28:857-59

12. Ding YH, Dai D, Lewis DA, et al. Intra-venous digital subtraction angiography (IVDSA): an alternative method to intra-arterial digital subtraction angiography (IADSA) for experimental aneurysm imaging. Neuroradiology 2005;47:792-95. Epub 2005 Aug 25

13. Brinjikji W, Cloft HJ, Lanzino G, et al. Comparison of 2D digital subtraction angiography and $3 \mathrm{D}$ rotational angiography in the evaluation of dome-to-neck ratio. AJNR Am J Neuroradiol 2009;30:831-34

14. Chien A, Castro MA, Tateshima S, et al. Quantitative hemodynamic analysis of brain aneurysms at different locations. AJNR Am J Neuroradiol 2009;30:1507-12

15. Raymond J, Darsaut T, Salazkin I, et al. Mechanisms of occlusion and recanalization in canine carotid bifurcation aneurysms embolized with platinum coils: an alternative concept. AJNR Am J Neuroradiol 2008;29:745-52

16. Killer M, Kallmes DF, McCoy MR, et al. Angiographic and histologic comparison of experimental aneurysms embolized with hydrogel filaments. AJNR Am J Neuroradiol 2009;30:1488-95. Epub 2009 May 27

17. Meng H, Wang ZJ, Hoi YM, et al. Complex hemodynamics at the apex of an arterial bifurcation induces vascular remodeling resembling cerebral aneurysm initiation. Stroke 2007;38:1924-31

18. Cloft HJ, Altes TA, Marx WF, et al. Endovascular creation of an in vivo bifurcation aneurysm model in rabbits. Radiology 1999;213: $223-28$ 